\title{
Thyroid hormones and functional outcomes after ischemic stroke
}

\author{
Lena M. O'Keefe', Sarah E. Conway², Alexandra Czap', Carl D. Malchoff', Sharon Benashski', Gilbert Fortunato², \\ llene Staff ${ }^{2}$ and Louise D. McCullough ${ }^{1,2^{*}}$
}

\begin{abstract}
Background: Stroke is the fifth leading cause of death and the primary cause of long-term adult disability in the United States. Increasing evidence suggests that low T3 levels immediately following acute ischemic stroke are associated with greater stroke severity, higher mortality rates, and poorer functional outcomes. Prognosis is also poor in critically ill hospitalized patients who have non-thyroidal illness syndrome (NTIS), where T3 levels are low, but TSH is normal. However, data regarding the association between TSH levels and functional outcomes are contradictory. Thus, this study investigated the role of TSH on stroke outcomes, concomitantly with T3 and T4.
\end{abstract}

Findings: In this work, blood was collected from patients with radiologically confirmed acute ischemic stroke at 24 \pm 6 hours post-symptom onset and serum levels of TSH, free T3, and free T4 were measured. Stroke outcomes were measured at discharge, 3 and 12 months using the modified Rankin scale and modified Barthel Index as markers of disability. Though we found that lower levels of free T3 were associated with worse prognosis at hospital discharge, and at 3 and 12 months post-stroke, none of these outcomes held after multivariate analysis. Thus, it is likely that thyroid hormones are associated with other factors that impact stroke outcomes, such as sex, age and stroke etiology.

Conclusions: This study found that lower levels of free T3 were associated with poorer outcomes at hospital discharge, and at 3 and 12 months post stroke, however, these associations diminished after correction for other known predictors of stroke outcome. Thyroid hormones have a complex relationship with ischemic stroke and stroke recovery, which merits further larger investigations.

Keywords: Thyroid hormones, Ischemic stroke, Stroke outcomes

\section{Introduction}

Stroke is the fifth leading cause of death and the primary cause of long-term adult disability in the United States [1]. Perturbations in the hypothalamus-pituitary-thyroid (HPT) axis affect stroke risk and stroke outcomes. Hypothyroidism can cause hypertension, hypercholesterolemia, cardiac dysfunction, and both hypo- and hypercoagulability, all of which are risk factors for stroke [2-4]. Hyperthyroidism is also associated with atrial fibrillation, which is a common cause of cardioembolic stroke [5].

The relationship between thyroid hormones and functional outcomes post-stroke is complex. Current data has shown that low T3 levels immediately following

\footnotetext{
* Correspondence: Imccullough@uchc.edu

${ }^{1}$ Department of Neuroscience, University of Connecticut Health Center, 263

Farmington Avenue, Farmington, CT 06030, USA

${ }^{2}$ Hartford Hospital, 80 Seymour Street, Hartford, CT, USA
}

acute ischemic stroke (AIS) are associated with greater stroke severity and mortality, and poorer functional outcomes [6-8]. This is also true in critically ill hospitalized patients who have non-thyroidal illness syndrome (NTIS; or 'euthyroid sick syndrome'), where T3 levels are low, but TSH is normal [9-11]. NTIS patients have poorer short-term prognosis and higher mortality rates at 12 months compared to non-NTIS patients [6]. However, data regarding the association between TSH levels and functional outcomes after stroke are conflicting [12-15]. The association between TSH levels and stroke outcome remains unclear, in part, because few studies have looked at TSH, T3, and T4 values concurrently. Doing so may be especially relevant in patients with brain injury and perturbations in HPT signaling.

This study examines the association between thyroid hormone levels (T3, T4, and TSH) and ischemic stroke 
outcomes as determined by the modified Rankin scale (mRS) and modified Barthel index (mBI) at hospital discharge, and at 3 and 12 months.

\section{Methods}

\section{Study setting and population}

This study was conducted at an 868-bed communitybased teaching hospital with a Neurology Residency Program. This center has The Joint Commission's Gold Seal of Approval and the American Heart Association's Advanced certification as a comprehensive stroke center and has an annual stroke admission rate of approximately 1000. This study was approved by the Institutional Review Board.

\section{Study protocol}

Blood was collected from patients over 18 years of age with radiologically confirmed acute ischemic stroke (AIS) $(n=129)$ at $24 \pm 6$ h post-symptom onset under an approved IRB from December 2011 to May 2013. Patients with active malignancy or treatment with immunosuppressants were excluded from the study. Serum levels of TSH, free T3 (fT3), and free T4 (fT4) were quantified by solid-phase chemiluminescent immunometric assay (R\&D Systems, Minneapolis, MN).

\section{Measurements}

Trained nursing staff prospectively collected clinical and patient information and entered it into the Stroke Center's database, which contains information regarding patient presentation, stroke etiology, and outcome. Baseline demographic information (age, sex, medical history, medication use) was collected. Risk factors such as history of hypertension, atrial fibrillation, coronary artery disease, previous stroke or transient ischemic attack, diabetes mellitus, hypercholesterolemia, cigarette smoking and alcohol use were also collected. Primary outcomes were inhospital mortality and National Institutes of Health Stroke Scale (NIHSS) score on admission. Secondary outcomes included death or hospice after discharge, and modified Rankin scale (mRS) and modified Barthel Index (mBI), at 3 and 12 months post-stroke. The mRS is a scale for measuring the disability of patients post-stroke. It has a scale of $0-6$, with 0 designating no limitations or symptoms, 5 being severe disability requiring constant care, and 6 being dead [16]. The $\mathrm{mBI}$ is a scale used to measure performance in activities of daily living. It is scored on a scale of $0-20$ with 20 being independent and 0 being completely dependent [17].

\section{Statistical analysis}

Continuous data, such as age, are presented as means (standard deviation). Non-normally distributed continuous and ordinal data are presented as medians (interquartile range) and were analyzed with nonparametric tests (Spearman's Rho). Categorical data are presented as proportions, and group differences were assessed with chi-square tests of proportion. Several variables with non-normal distributions were dichotomized and analyzed with chi-square test of proportions. These included the $\mathrm{mBI}$, dichotomized into "independent" (score of 15 or greater), or "dependent" (14 or less), and mRS, dichotomized into "disabled" (score of 3 or greater) or no disability to slight disability, termed "abled" (2 or less). A "poor outcome" composite measure was defined as death or disabled with an $\mathrm{mBI} \leq 14$ or a $m R S \geq 3$ as defined above at 3 and 12 months after AIS. A "favorable outcome" composite measure was defined as $m B I \geq 15$ or $m R S \leq 2$. Significant findings identified in the univariate analyses that pertained to more than one thyroid marker were reanalyzed using multivariable logistic regression to control for all significant confounding variables. Thus, a unified list of predictive factors was applied to all three thyroid markers in multivariate analysis. This included atrial fibrillation (AFIB), depression, urinary tract infection (UTI), intraarterial devices (IA), age and NIHSS on admission. The criterion of statistical significance was set at 0.05. All analyses were performed using Statistical Package for the Social Science v14.

\section{Results}

\section{Demographics}

Patient demographics are listed in Table 1. Out of the 129 patients, 119 were classified as euthyroid (TSH between 0.27 and 4.20 microunits/L), 7 as hyperthyroid ( $\mathrm{TSH}<0.27$ microunits $/ \mathrm{L})$, and 3 as hypothyroid $(\mathrm{TSH}>$ 4.20 microunits/L).

\section{Demographic associations}

As expected TSH was inversely correlated with fT4, though there was no significant correlation between TSH and fT3. fT3 and age were inversely correlated, but there was no significant association with age for TSH or fT4. There was no significant difference between males and females in TSH, fT4, or fT3 levels (Table 2).

\section{Pre-stroke associations}

Patients with low levels of fT3 had increased disability at baseline indicated by higher pre-stroke mRS scores $(r=-0.393, p<0.001)$ and lower mBI scores $(r=0.295$, $p=0.002)$ (Table 2).

\section{Admission}

The NIHSS on admission was inversely associated with fT3 $(R=-.191, p=0.031)$, but not TSH or fT4. Hospital mRS was inversely associated with TSH $(R=-0.206, p=$ $0.019)$, and directly associated with fT4 $(R=0.176, p=$ 
Table 1 Characteristics of study patients

\begin{tabular}{|c|c|}
\hline Category & \\
\hline Age $(y \pm s d)$ & $67.03 \pm 14.474$ \\
\hline \multicolumn{2}{|l|}{ Sex } \\
\hline Female & $51(39.5 \%)$ \\
\hline Male & $78(60.5 \%)$ \\
\hline \multicolumn{2}{|l|}{ Ethnicity } \\
\hline White & 99 (76.7 \%) \\
\hline Black & $12(9.3 \%)$ \\
\hline Latino & $15(11.6 \%)$ \\
\hline Other & $3(2.3 \%)$ \\
\hline \multicolumn{2}{|l|}{ History of } \\
\hline HTN & 106 (82.2 \%) \\
\hline Diabetes & 46 (35.7\%) \\
\hline Heart disease & 44 (34.1\%) \\
\hline Stroke & 19 (14.7\%) \\
\hline High cholesterol & $91(70.5 \%)$ \\
\hline Atrial fibrillation & 31 (24 \%) \\
\hline Angina & 0 \\
\hline Blood clots & $4(3.1 \%)$ \\
\hline Arthritis & 16 (12.4\%) \\
\hline Seizures & $5(3.9 \%)$ \\
\hline Dementia & $4(3.1 \%)$ \\
\hline Depression & 17 (13.2 \%) \\
\hline Lung problems & $8(6.2 \%)$ \\
\hline Aspiration pneumonia & $9(7.0 \%)$ \\
\hline Aneurysm & 0 \\
\hline UTI & $20(15.5 \%)$ \\
\hline Smoking & 25 (19.4\%) \\
\hline Alcohol & $5(3.9 \%)$ \\
\hline Drugs & $2(1.6 \%)$ \\
\hline \multicolumn{2}{|l|}{ Origin Location } \\
\hline Home & $61(4.3 \%)$ \\
\hline Community & 16 (12.4\%) \\
\hline Inpatient & $1(0.8 \%)$ \\
\hline Transfer patient & $44(34.1 \%)$ \\
\hline Facility & $6(4.7 \%)$ \\
\hline
\end{tabular}

Characteristics of patients enrolled in this study including demographics, past medical history, and origin location

HTN hypertension, UTI urinary tract infection

0.045). There was no association with fT3. Admission $\mathrm{mBI}$ score was unrelated to fT3, fT4, or TSH levels (Table 2).

\section{Outcome at discharge}

Patients with lower TSH had significantly higher in hospital mortality $(p=0.015)$. There was no association between hospital mortality and fT3 or fT4. However,
Table 2 Associations between thyroid hormones and patient demographics, baseline function, stroke severity, medical history, and medication usage

\begin{tabular}{|c|c|c|c|}
\hline & $\mathrm{TSH}$ & fT3 & fT4 \\
\hline \multicolumn{4}{|l|}{ Demographic associations } \\
\hline TSH & NS & NS & $R=-0.246^{*}$ \\
\hline Sex & NS & NS & NS \\
\hline Age & NS & $R=-.332^{* * *}$ & NS \\
\hline \multicolumn{4}{|l|}{ Pre-stroke associations } \\
\hline Baseline mRS & NS & $R=-0.393^{* * *}$ & NS \\
\hline Baseline mBI & NS & $R=0.295^{* * *}$ & NS \\
\hline \multicolumn{4}{|l|}{ Admission associations } \\
\hline Admission $\mathrm{NIH}$ & NS & $R=-0.191^{*}$ & NS \\
\hline Hospital mRS & $R=-0.206$ & NS & $R=0.176^{*}$ \\
\hline Admission mBl & NS & NS & NS \\
\hline \multicolumn{4}{|l|}{ Other associations } \\
\hline \multicolumn{4}{|l|}{ Past medical history } \\
\hline Hypertension & NS & NS & NS \\
\hline Heart disease & NS & NS & NS \\
\hline Prior stroke & NS & NS & NS \\
\hline Diabetes & NS & NS & NS \\
\hline Depression & NS & NS & NS \\
\hline Alcohol use & NS & NS & NS \\
\hline \multicolumn{4}{|l|}{ Medications } \\
\hline Anti-platelets & NS & NS & NS \\
\hline Anticoagulants & NS & NS & NS \\
\hline Anti-hypertensives & NS & NS & NS \\
\hline Anti-cholesterol & NS & NS & NS \\
\hline
\end{tabular}

Associations between thyroid hormones and demographics upon hospital admission. Patients with low levels of fT3 had increased disability and poorer pre-stroke function. NIH score on admission was inversely associated with fT3. Hospital mRS was inversely associated with TSH and directly associated with fT4. None of the thyroid hormones were associated with past medical history of hypertension, heart disease, prior stroke, diabetes, depression, alcohol use, or with the usage of anti-platelets, anti-coagulants, anti-hypertensives, or anti-cholesterol medications ${ }^{*} p<0.05 ;{ }^{* *} p<0.01 ;{ }^{* * *} p<0.001$

patients who were alive at discharge had significantly higher fT3 levels than patients who either died in hospital or went to hospice $(p=0.01)$ (Table 3).

\section{3 month outcomes}

Low TSH was related to 3 month mortality $(p=0.036)$. Patients who died at 3 months had lower TSH values than those who were alive at 3 months. Higher 24 h fT3 levels were predictive of good outcome at 3 months as measured by $\mathrm{mBI}>15(p=0.004)$. In addition, patients with a favorable composite outcome at 3 months had higher TSH levels than those with a poor composite outcome (Table 3). There was no association between outcome and fT4 (Table 3). 
Table 3 Outcomes

\begin{tabular}{|c|c|c|c|}
\hline & TSH & fT3 & fT4 \\
\hline \multicolumn{4}{|l|}{ Discharge outcomes } \\
\hline Death in hospital & & NS & NS \\
\hline Died & $0.5245(0.303-0.761)^{*}$ & & \\
\hline Alive & $1.275(0.75-1.9875)^{*}$ & & \\
\hline Death in hospital or hospice vs alive & NS & & NS \\
\hline Death or hospice & & $1.99(1.74-2.475)^{*}$ & \\
\hline Alive & & $2.47(2.13-2.95)^{*}$ & \\
\hline \multicolumn{4}{|l|}{3 month outcomes } \\
\hline Death vs alive at 3 months & & NS & NS \\
\hline Death & $0.66(0.372-1.45)^{*}$ & & \\
\hline Alive & $1.27(0.75-2.11)^{*}$ & & \\
\hline Independence level & NS & & NS \\
\hline $\mathrm{mBI}>15$, independent & & $2.53(2.24-2.99)^{* *}$ & \\
\hline $\mathrm{mBI}<14$, dependent & & $2.06(1.85-2.6)^{* *}$ & \\
\hline Poor outcome, mRS $>3$ or death & $0.873(0.586-1.48)^{*}$ & & \\
\hline Favourable outcome, mRS $<2$ & $1.54(0.6575-2.255)^{*}$ & & \\
\hline \multicolumn{4}{|l|}{12 month outcomes } \\
\hline mRS score & NS & $R=-0.268^{*}$ & NS \\
\hline $\mathrm{mBI}>15$, independent & & $2.525(2.26-2.965)^{*}$ & \\
\hline $\mathrm{mBl}<14$, dependent & & $2.175(1.865-2.823)^{*}$ & \\
\hline Poor outcome, mRS $>3$ or death & & $2.04(1.825-2.485)^{* * *}$ & \\
\hline Favourable outcome, mRS $<2$ & & $2.62(2.32-2.97)^{* * *}$ & \\
\hline
\end{tabular}

Patients with better acute and long term outcomes had higher TSH levels, and higher fT3 levels at 3 and 12 months post-stroke. No associations were seen between acute and long term outcomes with fT4

${ }^{*} p<0.05 ;{ }^{* *} p<0.01 ;{ }^{* * *} p<0.001$

\section{2 month outcomes}

The same pattern was seen at 12 months. Patients with the highest fT3 levels were the most likely to be independent as measured by the mBI 1 year after ischemic stroke $(p=0.042)$. This was confirmed by an inverse association between mRS and fT3 levels, showing that the higher the initial fT3, the better the outcome, even a year after the index event $(p=0.042)$. Neither $\mathrm{mBI}$ nor the mRS at 12 months was related to TSH or fT4 levels (Table 3).

\section{Other associations}

There were differences in fT3 based on stroke etiology determined with the TOAST classification. Patients with small vessel disease had higher fT3 values than those with large vessel or cardioembolic stroke etiology $(\mathrm{H}(2)=11.06$, $p=0.004$ ) with a mean rank of 83 for small vessel disease, 59 for large vessel disease, and 53 for cardioembolic stroke etiology.

\section{Multivariate}

A multivariate logistic regression model was used to control for the potential confounders identified in the univariate analyses as described above. When controlling for AFIB, depression, UTI, IA, age and NIH on admission, thyroid markers were not associated with mortality or functional outcomes (Table 4), suggesting that thyroid status is related to other risk factors rather than an independent predictor of outcome.

Table 4 Multivariate analysis

\begin{tabular}{|c|c|c|c|}
\hline & TSH: OR $(95 \% \mathrm{Cl}), \mathrm{P}$ & fT3: OR (95\% Cl), P & fT4: OR (95\% Cl), P \\
\hline Death in hospital or hospice & $0.635(0.250-1.613), \mathrm{NS}$ & $1.06(0.569-1.974), \mathrm{NS}$ & $1.957(0.323-11.872), \mathrm{NS}$ \\
\hline 3 month outcome, $\mathrm{mRS}>3$ or death vs $\mathrm{mRS}<2$ & $0.686(0.398-1.181), \mathrm{NS}$ & $0.517(0.226-1.185), \mathrm{NS}$ & $1.574(0.465-5.328), \mathrm{NS}$ \\
\hline 3 month outcome, $\mathrm{mBl}>15 \mathrm{vs} \mathrm{mBl}<14$ or death & $0.707(0.443-1.127), \mathrm{NS}$ & $0.513(.215-1.225), \mathrm{NS}$ & \\
\hline
\end{tabular}




\section{Discussion}

This study is the first to look at TSH, fT3, and fT4 concurrently and their association with stroke severity and long term functional outcomes. This study found that low TSH and $\mathrm{fT} 3$ were associated with poor function at 3 months, and that low TSH and fT3 were associated with higher rates of death in the hospital. However, these results were not seen in the multivariate analysis when other known predictors of outcome such as stroke severity (NIHSS) were controlled. This is likely due to the relatively small sample size of the study, and the fact that the majority of patients sampled were euthyroid. Though prior studies have demonstrated an association between low T3 levels and poor outcomes, it is likely that this study was underpowered to detect an association. Outcomes at 12 months were only available in a minority of patients. Future studies with an increased number of patients with hyper- and hypothyroidism and hypothyroidism with hormone replacement should be done to assess a wider range of thyroid values and outcomes. In addition, assessment of hormone levels over multiple time points is needed to demonstrate changes in thyroid status over time, as only one time-point was evaluated here. However, the significant association between thyroid hormone levels and outcome in the univariate models suggest that this is worth pursuing vigorously in larger studies, as strategies to replace thyroid levels could be developed for therapeutic trials.

Although it is unknown whether administration of exogenous thyroid hormone in stroke patients improves outcomes, administration of $\mathrm{T} 3$ resulted in increased mRNA expression of reelin and brain-derived neurotrophic factor in rats, both of which are important in nervous system regeneration [18]. In hospital patient monitoring of thyroid status may be important as higher levels of fT3 have been associated with better functional outcomes. Therefore, treatment to normalize and/or elevate fT3 values for patients presenting with NTIS could be beneficial to a patient's recovery after acute ischemic stroke.

\footnotetext{
Abbreviations

HPT: Hypothalamus-pituitary-thyroid axis; AIS: Acute ischemic stroke; NTIS: Non-thyroidal illness syndrome; mRS: Modified Rankin scale; mBI: Modified Barthel index; NIHSS: National Institutes of Health Stroke Scale; AFIB: Atrial fibrillation; UTI: Urinary tract infection; IA: Intra-arterial devices; fT3: Free T3; fT4: Free T4.
}

\section{Competing interests}

The authors declare that they have no competing interests.

\section{Authors' contributions}

LMO and SEC consented patients, collected blood samples, conducted 3 and 12 month modified Barthel index and modified Rankin scale questionnaires (hereafter referred to as 'collected data'), and drafted the manuscript. AC contributed to the study design and collected data. CDM contributed to the study design. SB collected data and conducted the enzyme-linked immunoadsorption assay. GF compiled patient demographics and data. IS coordinated all statistical analyses. LDM contributed to the study design, collected data, and made all final decisions regarding the manuscript. All authors read and approved the final manuscript.
Acknowledgements

Pamela Fall in the General Clinical Research Center for running the ELISAs.

\section{Grant support}

Hartford Hospital Research Endowment Funds, Hartford Hospital and The Lowell P. Weicker, Jr. Clinical Research Center, University of Connecticut Health Center.

Received: 1 June 2015 Accepted: 10 June 2015

Published online: 04 July 2015

\section{References}

1. Go AS, Mozaffarian D, Roger VL, et al. Heart Disease and Stroke Statistics -2014 Update: A Report from the American Heart Association. 2014. p. e28-292. doi:10.1161/01.cir.0000441139.02102.80.

2. Gao N, Zhang W, Zhang Y, Yang Q, Chen S. Carotid intima-media thickness in patients with subclinical hypothyroidism: a meta-analysis. Atherosclerosis. 2013;227:18-25. doi:10.1016/j.atherosclerosis.2012.10.070.

3. Iwen KA, Schröder E, Brabant G. Thyroid Hormones and the Metabolic Syndrome. Eur Thyroid J. 2013;2:83-92. doi:10.1159/000351249.

4. Bai M-F, Gao C-Y, Yang C-K, et al. Effects of thyroid dysfunction on the severity of coronary artery lesions and its prognosis. J Cardiol. 2014;64(6):496-500. doi:10.1016/j.jjcc.2014.03.009.

5. Chen Q, Yan Y, Zhang L, Cheng K, Liu Y, Zhu W. Effect of hyperthyroidism on the hypercoagulable state and thromboembolic events in patients with atrial fibrillation. J Cardiol. 2014;127:176-82. doi:10.1159/000356954.

6. Alevizaki M, Synetou M, Xynos K, Pappa T, Vemmos KN. Low triiodothyronine: A strong predictor of outcome in acute stroke patients. Eur J Clin Invest. 2007;37:651-7. doi:10.1111/j.1365-2362.2007.01839.x.

7. Zhang Y, Meyer MA. Clinical analysis on alteration of thyroid hormones in the serum of patients with acute ischemic stroke. Stroke Res Treat. 2010;2010. doi:10.4061/2010/290678.

8. Bunevicius A, lervasi G, Bunevicius R. Neuroprotective actions of thyroid hormones and low-T3 syndrome as a biomarker in acute cerebrovascular disorders. Expert Rev Neurother. 2015;15(3):315-26.

9. Chopra IJ. Clinical review 86: Euthyroid sick syndrome: is it a misnomer? J Clin Endocrinol Metab. 1997;82:329-34. doi:10.1210/jc.82.2.329.

10. Mclver B, Gorman CA. Euthyroid sick syndrome: an overview. Thyroid. 1997;7:125-32. doi:10.1089/thy.1997.7.125.

11. Van den Berghe G, de Zegher F, Bouillon R. Clinical review 95: Acute and prolonged critical illness as different neuroendocrine paradigms. J Clin Endocrinol Metab. 1998;83:1827-34.

12. Akhoundi FH, Ghorbani A, Soltani A, Meysamie A. Favorable functional outcomes in acute ischemic stroke patients with subclinical hypothyroidism. Neurology. 2011;77:349-54. doi:10.1212/WNL.0b013e3182267ba0.

13. Wollenweber FA, Zietemann V, Gschwendtner A, Opherk C, Dichgans M. Subclinical hyperthyroidism is a risk factor for poor functional outcome after ischemic stroke. Stroke. 2013;44:1446-8. doi:10.1161/STROKEAHA.113.000833.

14. Alevizaki M, Synetou M, Xynos K, Alevizaki CC, Vemmos KN. Hypothyroidism as a protective factor in acute stroke patients. Clin Endocrinol (Oxf). 2006;65:369-72. doi:10.1111/j.1365-2265.2006.02606.x.

15. Leonards CO, Schneider HJ, Liman TG, Fiebach JB, Endres M, Ebinger M. Thyroid-stimulating hormone, white matter hyperintensities, and functional outcome in acute ischemic stroke patients. Cerebrovasc Dis Extra. 2014;4:61-8. doi:10.1159/000360217.

16. Van Swieten JC, Koudstaal PJ, Visser MC, Schouten HJ, van Gijn J. Interobserver agreement for the assessment of handicap in stroke patients. Stroke. 1988;19:604-7. doi:10.1161/01.STR.20.6.828.

17. Shah S, Vanclay F, Cooper B. Improving the sensitivity of the Barthel Index for stroke rehabilitation. J Clin Epidemiol. 1989;42:703-9. doi:10.1016/08954356(89)90065-6.

18. Sui L, Ren W-W, Li B-M. Administration of thyroid hormone increases reelin and brain-derived neurotrophic factor expression in rat hippocampus in vivo. Brain Res. 2010;1313:9-24. doi:10.1016/j.brainres.2009.12.010. 